\title{
Rigorous Solutions of Large-Scale Dielectric Problems with the Parallel Multilevel Fast Multipole Algorithm
}

\author{
Özgür Ergül ${ }^{1}$ and Levent Gürel ${ }^{2,3}$ \\ ${ }^{1}$ Department of Mathematics and Statistics \\ University of Strathclyde, Glasgow, UK \\ ${ }^{2}$ Department of Electrical and Electronics Engineering \\ ${ }^{3}$ Computational Electromagnetics Research Center (BiLCEM) \\ Bilkent University, Bilkent, Ankara, Turkey \\ Email: ozgur.ergul@strath.ac.uk, lgurel@bilkent.edu.tr
}

\begin{abstract}
We present fast and accurate solutions of large-scale electromagnetics problems involving three-dimensional homogeneous dielectric objects. Problems are formulated rigorously with the electric and magnetic current combined-field integral equation (JMCFIE) and solved iteratively with the multilevel fast multipole algorithm (MLFMA). In order to solve large-scale problems, MLFMA is parallelized efficiently on distributedmemory architectures using the hierarchical partitioning strategy. Efficiency and accuracy of the developed implementation are demonstrated on very large scattering problems discretized with tens of millions of unknowns.
\end{abstract}

\section{Introduction}

Real-life electromagnetics problems often involve big objects, whose accurate discretizations lead to very large matrix equations involving millions of unknowns. Such large matrix equations can be solved iteratively with the multilevel fast multipole algorithm (MLFMA) in $\mathcal{O}(N \log N)$ time using $\mathcal{O}(N \log N)$ memory, where $N$ is the number of unknowns [1]. Since MLFMA running on a single processor may not be sufficient to solve large-scale problems in reasonable times, it is essential to parallelize and employ MLFMA on clusters of processors [2]-[9]. Parallelization of MLFMA on distributed-memory architectures is particularly popular due to the relatively inexpensive costs of these architectures [2]-[6],[8],[9]. In fact, the last decade has seen enormous advances in this area, increasing the problem sizes from tens of millions to over one billion. On the other hand, parallel implementations have been developed mostly for analyzing perfectly conducting objects, and less attention has been paid to dielectric structures [5].

In this study, we consider the solution of electromagnetics problems involving three-dimensional dielectric objects with parallel MLFMA. For the efficient parallelization of MLFMA, we use a rigorous technique, namely, the hierarchical partitioning strategy, which was originally developed for perfectly conducting objects [6]. Using the hierarchical strategy for dielectric structures, tree structures associated with the outer and inner media are distributed optimally among the processors. The resulting implementation is used to solve large-scale problems discretized with tens of millions unknowns.

\section{Solutions of Integral-Equation Formulations with Parallel MLFMA}

Discretizations of surface integral equations for dielectric objects lead to $2 N \times 2 N$ dense matrix equations in the form of

$$
\left[\begin{array}{ll}
\overline{\boldsymbol{Z}}^{(11)} & \overline{\boldsymbol{Z}}^{(12)} \\
\overline{\boldsymbol{Z}}^{(21)} & \overline{\boldsymbol{Z}}^{(22)}
\end{array}\right] \cdot\left[\begin{array}{l}
\boldsymbol{x} \\
\boldsymbol{y}
\end{array}\right]=\left[\begin{array}{l}
\boldsymbol{v}^{(1)} \\
\boldsymbol{v}^{(2)}
\end{array}\right],
$$

where $\boldsymbol{x}$ and $\boldsymbol{y}$ are vectors of $N$ elements involving the coefficients for the equivalent electric and magnetic currents, respectively. Dielectric problems can be formulated rigorously with the electric and magnetic current combined-field integral equation (JMCFIE) [10]. For this formulation, matrix elements and the 
elements of the right-hand-side vectors are derived as

$$
\begin{aligned}
Z_{m n}^{(11)} & =Z_{m n}^{(22)}=\int_{S_{m}} d \boldsymbol{r} \boldsymbol{t}_{m}(\boldsymbol{r}) \cdot\left(\mathcal{T}_{o}+\mathcal{T}_{i}\right)\left\{\boldsymbol{b}_{n}\right\}(\boldsymbol{r})+\int_{S_{m}} d \boldsymbol{r} \boldsymbol{t}_{m}(\boldsymbol{r}) \cdot \hat{\boldsymbol{n}} \times\left(\mathcal{K}_{o}-\mathcal{K}_{i}\right)\left\{\boldsymbol{b}_{n}\right\}(\boldsymbol{r}) \\
& -\int_{S_{m}} d \boldsymbol{r} \boldsymbol{t}_{m}(\boldsymbol{r}) \cdot \boldsymbol{b}_{n}(\boldsymbol{r}), \\
Z_{m n}^{(12)} & =\int_{S_{m}} d \boldsymbol{r} \boldsymbol{t}_{m}(\boldsymbol{r}) \cdot \hat{\boldsymbol{n}} \times\left(\eta_{o}^{-1} \mathcal{T}_{o}-\eta_{i}^{-1} \mathcal{T}_{i}\right)\left\{\boldsymbol{b}_{n}\right\}(\boldsymbol{r})-\int_{S_{m}} d \boldsymbol{r} \boldsymbol{t}_{m}(\boldsymbol{r}) \cdot\left(\eta_{o}^{-1} \mathcal{K}_{o}+\eta_{i}^{-1} \mathcal{K}_{i}\right)\left\{\boldsymbol{b}_{n}\right\}(\boldsymbol{r}) \\
& -\frac{1}{2}\left(\eta_{o}^{-1}-\eta_{i}^{-1}\right) \int_{S_{m}} d \boldsymbol{r} \boldsymbol{t}_{m}(\boldsymbol{r}) \cdot \hat{\boldsymbol{n}} \times \boldsymbol{b}_{n}(\boldsymbol{r}), \\
Z_{m n}^{(21)} & =-\int_{S_{m}} d \boldsymbol{r} \boldsymbol{t}_{m}(\boldsymbol{r}) \cdot \hat{\boldsymbol{n}} \times\left(\eta_{o} \mathcal{T}_{o}-\eta_{i} \mathcal{T}_{i}\right)\left\{\boldsymbol{b}_{n}\right\}(\boldsymbol{r})+\int_{S_{m}} d \boldsymbol{r} \boldsymbol{t}_{m}(\boldsymbol{r}) \cdot\left(\eta_{o} \mathcal{K}_{o}+\eta_{i} \mathcal{K}_{i}\right)\left\{\boldsymbol{b}_{n}\right\}(\boldsymbol{r}) \\
& +\frac{1}{2}\left(\eta_{o}-\eta_{i}\right) \int_{S_{m}} d \boldsymbol{r} \boldsymbol{t}_{m}(\boldsymbol{r}) \cdot \hat{\boldsymbol{n}} \times \boldsymbol{b}_{n}(\boldsymbol{r}), \\
v_{m}^{(1)} & =-\int_{S_{m}} d \boldsymbol{r} \boldsymbol{t}_{m}(\boldsymbol{r}) \cdot \hat{\boldsymbol{n}} \times \boldsymbol{H}^{i n c}(\boldsymbol{r})-\eta_{o}^{-1} \int_{S_{m}} d \boldsymbol{r} \boldsymbol{t}_{m}(\boldsymbol{r}) \cdot \boldsymbol{E}^{i n c}(\boldsymbol{r}), \\
v_{m}^{(2)} & =\int_{S_{m}} d \boldsymbol{r} \boldsymbol{t}_{m}(\boldsymbol{r}) \cdot \hat{\boldsymbol{n}}(\boldsymbol{r}) \times \boldsymbol{E}^{i n c}(\boldsymbol{r})-\eta_{o} \int_{S_{m}} d \boldsymbol{r} \boldsymbol{t}_{m}(\boldsymbol{r}) \cdot \boldsymbol{H}^{i n c}(\boldsymbol{r}),
\end{aligned}
$$

where the integro-differential operators are defined for the outer $(u=o)$ and inner $(u=i)$ media as

$$
\begin{aligned}
& \mathcal{T}_{u}\left\{\boldsymbol{b}_{n}\right\}(\boldsymbol{r})=i k_{u} \int_{S_{n}} d \boldsymbol{r}^{\prime} \boldsymbol{b}_{n}\left(\boldsymbol{r}^{\prime}\right) g_{u}\left(\boldsymbol{r}, \boldsymbol{r}^{\prime}\right)+\frac{i}{k_{u}} \int_{S_{n}} d \boldsymbol{r}^{\prime} \nabla^{\prime} \cdot \boldsymbol{b}_{n}\left(\boldsymbol{r}^{\prime}\right) \nabla g_{u}\left(\boldsymbol{r}, \boldsymbol{r}^{\prime}\right), \\
& \mathcal{K}_{u}\left\{\boldsymbol{b}_{n}\right\}(\boldsymbol{r})=\int_{\mathrm{PV}, S_{n}} d \boldsymbol{r}^{\prime} \boldsymbol{b}_{n}\left(\boldsymbol{r}^{\prime}\right) \times \nabla^{\prime} g_{u}\left(\boldsymbol{r}, \boldsymbol{r}^{\prime}\right) .
\end{aligned}
$$

In (2)-(8), $\boldsymbol{t}_{m}(\boldsymbol{r})$ and $\boldsymbol{b}_{n}(\boldsymbol{r})$ represent the $m$ th testing function and the $n$th basis function with spatial supports of $S_{m}$ and $S_{n}$, respectively, $\hat{\boldsymbol{n}}$ is the unit normal vector at the testing point, $\eta_{u}=\left(\mu_{u} / \epsilon_{u}\right)^{1 / 2}$ is the intrinsic impedance, $k_{u}=\omega\left(\epsilon_{u} \mu_{u}\right)^{1 / 2}$ is the wavenumber, $\boldsymbol{E}^{i n c}$ and $\boldsymbol{H}^{i n c}$ are the electric and magnetic fields created by external sources in the outer medium extending to infinity, and $g_{u}\left(\boldsymbol{r}, \boldsymbol{r}^{\prime}\right)=\exp \left(i k_{u}\left|\boldsymbol{r}-\boldsymbol{r}^{\prime}\right|\right) /\left|\boldsymbol{r}-\boldsymbol{r}^{\prime}\right|$ denotes the homogeneous-space Green's function.

Matrix equation in (1) can be solved iteratively via a Krylov-subspace algorithm, where the required matrix-vector multiplications are performed efficiently with MLFMA [1]. Multilevel tree structures (one for each medium) are constructed by placing the object in a cubic box and recursively dividing the computational domain into subdomains. This way, interactions between the distant basis and testing functions are calculated in three stages, namely, aggregation, translation, and disaggregation. In the aggregation stage, radiated fields are calculated at subdomain centers from the bottom of the tree structure to the top level. In the translation stage, radiated fields are converted into incoming fields between the subdomains. Finally, in the disaggregation stage, total incoming fields are calculated from the top of the structure to the bottom level. Different tree structures are required for the outer and inner media, since radiated and incoming fields are sampled at a rate that depends on the wavenumber, hence the electrical parameters of the medium.

MLFMA can be parallelized efficiently using a hierarchical partitioning strategy [6], which is based on the simultaneous partitioning of both subdomains and their fields at each level of the tree structure. Specifically, for a parallelization among $p$ processes, subdomains and field samples are divided into $p_{s}$ and $p_{f}=p / p_{s}$ partitions, respectively, where $p_{s}$ and $p_{f}$ are determined by load-balancing algorithms. Changing the partitioning scheme between levels bears an additional cost, but in general, the hierarchical strategy improves the load-balancing and reduces the communication time, compared to other available strategies. In this study, the hierarchical strategy is applied for the first time to solve dielectric problems.

\section{$3 \quad$ Numerical Examples}

All solutions presented in this paper are performed on a cluster of Intel Xeon Nehalem quad-core processors with $2.80 \mathrm{GHz}$ clock rate. MLFMA is parallelized into 64 processes using the hierarchical strategy. 

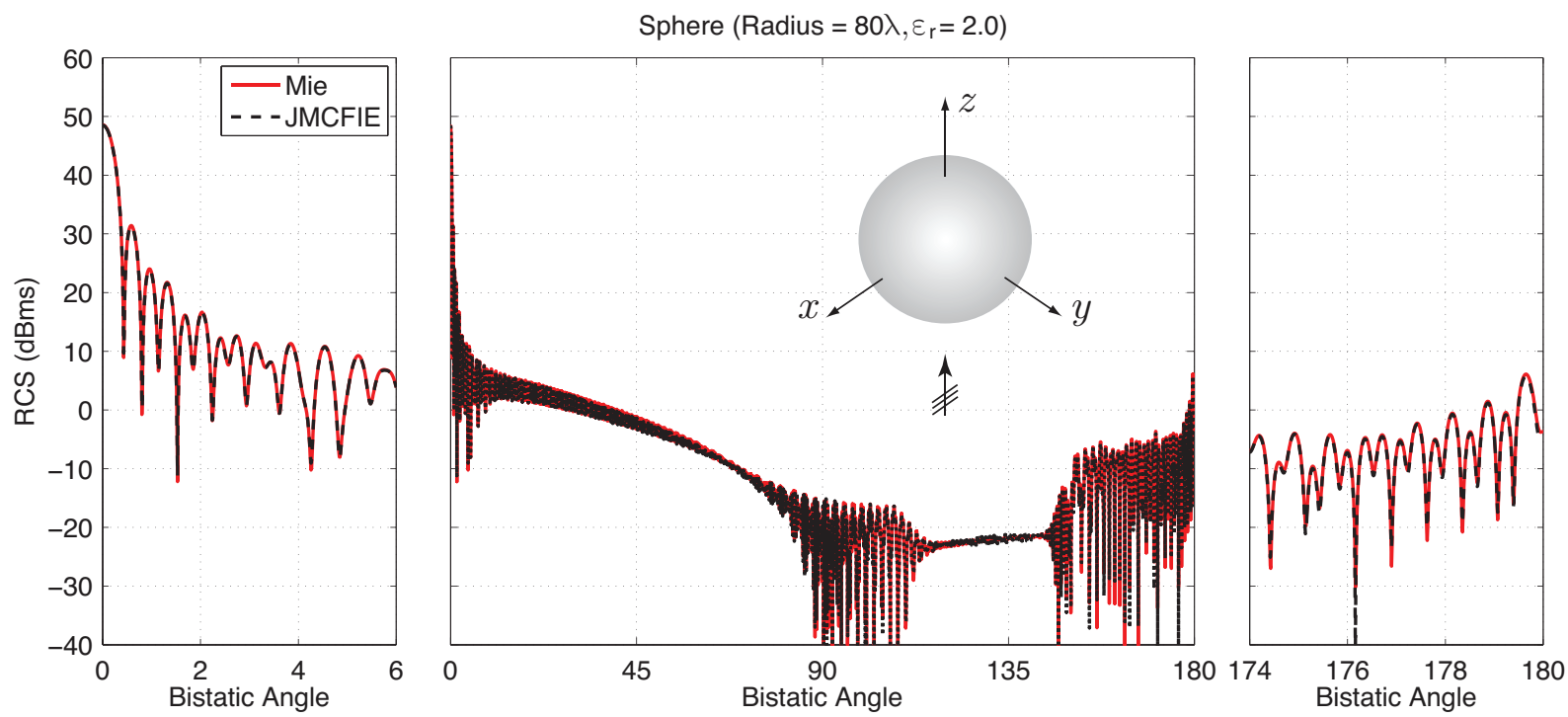

Figure 1: Solution of a scattering problem involving a dielectric sphere of radius $0.3 \mathrm{~m}$ at $80 \mathrm{GHz}$. The relative permittivity of the sphere is 2.0 . The total RCS is plotted as a function of bistatic angle from $0^{\circ}$ to $180^{\circ}$, where $0^{\circ}$ corresponds to the forward-scattering direction.

Discretizations are performed with the Rao-Wilton-Glisson functions on $\lambda / 10$ triangles, where $\lambda=2 \pi / k_{o}$ is the wavelength in the outer medium. Both near-field and far-field interactions are computed with maximum $1 \%$ error. Iterative solutions are performed with the biconjugate-gradient-stabilized (BiCGStab) algorithm and iterations are carried out until the residual error is reduced to below 0.005. Convergence of iterations is accelerated using block-diagonal preconditioners.

Fig. 1 presents the solution of a scattering problem involving a dielectric sphere of radius $0.3 \mathrm{~m}$ at $80 \mathrm{GHz}$ discretized with $46,811,328$ unknowns. The relative permittivity of the sphere is 2.0 and it is illuminated by a plane wave propagating in the $z$ direction with the electric field polarized in the $x$ direction in free space. The solution requires 107 iterations and the total time required for both setup and solution is 29 hours. Fig. 1 depicts the bistatic radar cross section (RCS) values (in $\mathrm{dB}$ meter square) on the $z-x$ plane as a function of the bistatic angle $\theta$ from $0^{\circ}$ to $180^{\circ}$. RCS values around the forward-scattering $\left(0^{\circ}\right)$ and back-scattering $\left(180^{\circ}\right)$ directions are focused in separate plots. We observe that computational values obtained with the parallel MLFMA perfectly agree with the analytical Mie-series results.

Fig. 2 presents the solution of a scattering problem involving a dielectric hemisphere lens with $25 \mathrm{~mm}$ radius. The lens has a relative permittivity of 4.8 and it is investigated at $120 \mathrm{GHz}$ and $960 \mathrm{GHz}$, corresponding to radii of $10 \lambda$ and $80 \lambda$, respectively. Discretizations at these frequencies lead to matrix equations involving 615,456 and 39,389,184 unknowns, respectively. At both frequencies, the lens is illuminated by a plane wave propagating in the $-z$ direction. Fig. 2(a) depicts the total electric field in the vicinity of the lens at $120 \mathrm{GHz}$, where focusing is clearly observed in the transmission region. Fig. 2(b) depicts the total electric field from $z=-40 \mathrm{~mm}$ to $40 \mathrm{~mm}$ at $960 \mathrm{GHz}$. Focusing is again observed at around $-9 \mathrm{~mm}$. For the larger problem, the number of iterations is 68 , whereas the total time is 22 hours.

\section{Conclusion}

This study presents rigorous solutions of large-scale electromagnetics problems involving homogeneous dielectric objects. Problems are formulated with JMCFIE and solved iteratively via MLFMA. For large-scale computations, MLFMA is parallelized using the hierarchical partitioning strategy. We show that dielectric problems discretized with tens of millions of unknowns can be solved accurately and efficiently with the developed implementation. 


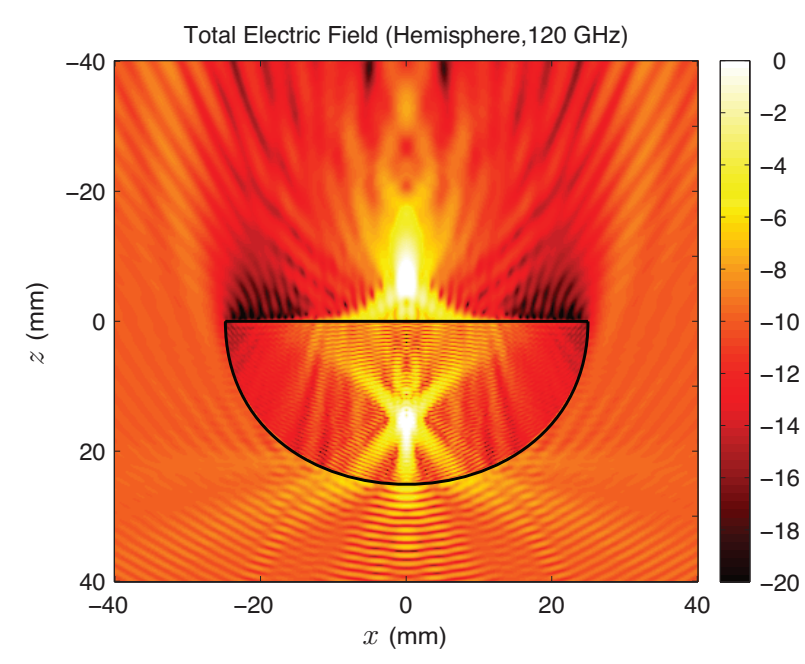

(a)

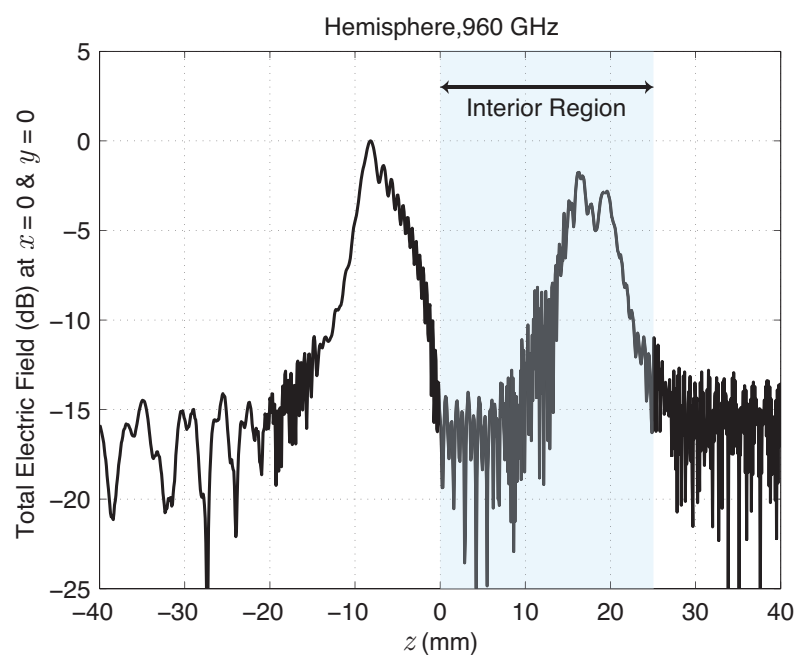

(b)

Figure 2: Solutions of scattering problems involving a dielectric hemisphere lens with 25 mm radius. The lens has a relative permittivity of 4.8. (a) The total electric field in the vicinity of the lens at $120 \mathrm{GHz}$. (b) The total electric field from $z=-40 \mathrm{~mm}$ to $40 \mathrm{~mm}$ at $960 \mathrm{GHz}$.

\section{Acknowledgments}

This work was supported by the Centre for Numerical Algorithms and Intelligent Software (EPSRC EP/G036136/1), the Scientific and Technical Research Council of Turkey (TUBITAK) under the Research Grant 110E268, by the Turkish Academy of Sciences in the framework of the Young Scientist Award Program (LG/TUBA-GEBIP/2002-1-12), and by contracts from ASELSAN and SSM. Özgür Ergül was also supported by a Research Starter Grant provided by the Faculty of Science at the University of Strathclyde.

\section{References}

1. J. Song, C.-C. Lu, and W. C. Chew, "Multilevel fast multipole algorithm for electromagnetic scattering by large complex objects," IEEE Trans. Antennas Propag., vol. 45, no. 10, pp. 1488-1493, Oct. 1997.

2. S. Velamparambil, W. C. Chew, and J. Song, "10 million unknowns: Is it that big?," IEEE Antennas Propag. Mag., vol. 45, no. 2, pp. 43-58, Apr. 2003.

3. L. Gürel and Ö. Ergül, "Fast and accurate solutions of integral-equation formulations discretised with tens of millions of unknowns," Electron. Lett., vol. 43, no. 9, pp. 499-500, Apr. 2007.

4. Ö. Ergül and L. Gürel, "Efficient parallelization of the multilevel fast multipole algorithm for the solution of large-scale scattering problems," IEEE Trans. Antennas Propag., vol. 56, no. 8, pp. 2335-2345, Aug. 2008. 5. J. Fostier and F. Olyslager, "An asynchronous parallel MLFMA for scattering at multiple dielectric objects," IEEE Trans. Antennas Propag., vol. 56, no. 8, pp. 2346-2355, Aug. 2008.

6. Ö. Ergül and L. Gürel, "A hierarchical partitioning strategy for an efficient parallelization of the multilevel fast multipole algorithm," IEEE Trans. Antennas Propag., vol. 57, no. 6, pp. 1740-1750, Jun. 2009.

7. J. M. Taboada, L. Landesa, F. Obelleiro, J. L. Rodriguez, J. M. Bertolo, M. G. Araujo, J. C. Mourino, and A. Gomez, "High scalability FMM-FFT electromagnetic solver for supercomputer systems," IEEE Antennas Propag. Mag., vol. 51, no. 6, pp. 21-28, Dec. 2009.

8. J. M. Taboada, M. G. Araujo, J. M. Bertolo, L. Landesa, F. Obelleiro, and J. L. Rodriguez, "MLFMAFFT parallel algorithm for the solution of large-scale problems in electromagnetics," Prog. Electromagn. Res., vol. 105, pp. 15-30, 2010.

9. Ö. Ergül and L. Gürel, "Rigorous solutions of electromagnetics problems involving hundreds of millions of unknowns," IEEE Antennas Propag. Mag., accepted for publication, 2011.

10. P. Ylä-Oijala and M. Taskinen, "Application of combined field integral equation for electromagnetic scattering by dielectric and composite objects," IEEE Trans. Antennas Propagat., vol. 53, no. 3, pp. 11681173, Mar. 2005. 